\title{
A Novel Risk Factor Associated With Colonization by Carbapenemase-Producing Enterobacteriaceae: Use of Proton Pump Inhibitors in Addition to Antimicrobial Treatment
}

\author{
Vincent C. C. Cheng, MD; ;,2 Jonathan H. K. Chen, PhD; ${ }^{1}$ Simon Y. C. So, MMedSc; ${ }^{1}$ Sally C. Y. Wong, FRCPath; ${ }^{1}$ \\ Pui-Hing Chau, PhD; ${ }^{3}$ Lisa M. W. Wong, MSc(IC); ${ }^{2}$ Radley H. C. Ching, BSN; ${ }^{2}$ Modissa M. L. Ng, MSN; ${ }^{2}$ \\ Wan-Mui Lee, MSN; ${ }^{2}$ Ivan F. N. Hung, MD; ${ }^{4}$ Pak-Leung Ho, MD; ${ }^{1}$ Kwok-Yung Yuen, MD $^{1}$
}

овјестіvе. To study the association between gastrointestinal colonization of carbapenemase-producing Enterobacteriaceae (CPE) and proton pump inhibitors (PPIs).

METHODs. We analyzed 31,526 patients with prospective collection of fecal specimens for CPE screening: upon admission (targeted screening) and during hospitalization (opportunistic screening, safety net screening, and extensive contact tracing), in our healthcare network with 3,200 beds from July 1, 2011, through December 31, 2015. Specimens were collected at least once weekly during hospitalization for CPE carriers and subjected to broth enrichment culture and multiplex polymerase chain reaction.

RESUlts. Of 66,672 fecal specimens collected, 345 specimens $(0.5 \%)$ from 100 patients $(0.3 \%)$ had CPE. The number and prevalence (per 100,000 patient-days) of CPE increased from $2(0.3)$ in 2012 to 63 (8.0) in 2015 ( $P<.001)$. Male sex (odds ratio, 1.91 [95\% CI, 1.15-3.18], $P=.013)$, presence of wound or drain (3.12 [1.70-5.71], $P<.001)$, and use of cephalosporins (3.06 [1.42-6.59], $P=.004)$, carbapenems (2.21 [1.10-4.48], $P=.027)$, and PPIs $(2.84[1.72-4.71], P<.001)$ in the preceding 6 months were significant risk factors by multivariable analysis. Of 79 patients with serial fecal specimens, spontaneous clearance of CPE was noted in 57 (72.2\%), with a median (range) of 30 (3-411) days. Comparing patients without use of antibiotics and PPIs, consumption of both antibiotics and PPIs after CPE identification was associated with later clearance of CPE (hazard ratio, 0.35 [95\% CI, 0.17-0.73], $P=.005$ ).

CONCLUSIONS. Concomitant use of antibiotics and PPIs prolonged duration of gastrointestinal colonization by CPE.

Infect Control Hosp Epidemiol 2016;3 7:1418-1425

Carbapenemase-producing Enterobacteriaceae (CPE) are emerging multidrug-resistant gram-negative organisms causing an unprecedented public health threat. ${ }^{1}$ Therapeutic options for invasive infections are limited and the mortality of bloodstream infection caused by CPE is up to $37 \%$. $^{2}$ The nature of plasmid-mediated carbapenemases such as class $\mathrm{A}$ enzymes of the KPC type, zinc-dependent class B metallo$\beta$-lactamases of mainly NDM, IMP, and VIM types, and class D carbapenemase of OXA-48 type facilitates global transmission and causes numerous outbreaks of CPE in the healthcare settings. $^{3-5}$ Failure in recognizing asymptomatic carriers may result in nosocomial transmission ${ }^{6}$ or even country-wide outbreak. ${ }^{7}$ Therefore, enhanced infection control measures such as active surveillance, isolation and cohorting of CPE patients with dedicated nursing staff and medical equipment, and daily bathing with chlorhexidine gluconate have been implemented to control the spread of CPE in the endemic settings. $^{8}$

Compared with the global epidemiology, CPE is not yet endemic in Hong Kong and mainland China. ${ }^{9,10}$ It is important to prevent importation and nosocomial transmission of CPE in our healthcare settings through the implementation of proactive infection control measures ${ }^{11}$ and to understand the risk factors for CPE acquisition, such as recent hospitalization and the presence of indwelling devices. ${ }^{12}$ Here, we analyzed the risk factors and duration of CPE colonization among the first 100 patients with gastrointestinal colonization of CPE in our healthcare network. Unexpectedly, the use of proton pump inhibitors (PPIs) was independently associated with fecal colonization and carriage duration of CPE. The finding is important for the formulation of infection control policy.

Affiliations: 1. Department of Microbiology, Queen Mary Hospital, Hong Kong Special Administrative Region, China; 2. Infection Control Team, Queen Mary Hospital, Hong Kong Special Administrative Region, China; 3. School of Nursing, The University of Hong Kong, Hong Kong Special Administrative Region, China; 4. Department of Medicine, Queen Mary Hospital, Hong Kong Special Administrative Region, China.

Received May 27, 2016; accepted August 4, 2016; electronically published September 13, 2016 (c) 2016 by The Society for Healthcare Epidemiology of America. All rights reserved. 0899-823X/2016/3712-0004. DOI: 10.1017/ice.2016.202 


\section{METHODS}

\section{Setting}

As part of the routine hospital surveillance to control CPE, we analyzed the microbiologic and epidemiologic findings of gastrointestinal colonization of carbapenem-resistant Enterobacteriaceae (CRE) and CPE in a healthcare network (Hong Kong West Hospital Network) that comprises an acute care university-affiliated teaching hospital with 1,600 beds as well as 3 extended-care hospitals with another 1,600 beds in Hong Kong. According to our infection control program, patients were prospectively screened for CPE in target admission screening, opportunistic screening, safety net screening, and extensive contact tracing during hospitalization as previously described. ${ }^{11,13,14}$ The duration of gastrointestinal colonization was monitored in patients colonized with CPE by surveillance fecal specimens collected at least once weekly, or more frequently if clinically indicated. The duration of carriage was taken as the date of the first positive to the date of $\mathrm{CPE}$ clearance. Clearance of $\mathrm{CPE}$ was assumed if a patient had at least 2 consecutive negative cultures taken 48 hours apart. To ensure fecal specimens were collected from all patients meeting the criteria, an electronic file of patient data was automatically generated by the hospital record office and was passed onto the infection control team on each working day. Newly admitted patients who fulfilled the screening criteria and those patients who had been hospitalized for 14 days were highlighted in the electronic file, which was further compared with the registration data of fecal specimens received for CPE screening in the laboratory information system in the microbiology laboratory. For patients who met the CPE screening criteria but had no fecal specimen received by the laboratory according to the system, the infection control nurses would remind the frontline staff for specimen collections. Usually, rectal swabs were collected for targeted screening, safety net screening, and extensive contact tracing, whereas stool specimens requested for Clostridium difficile were screened routinely for CPE (opportunistic screening).

Upon identification of gastrointestinal colonization of CPE, infection control nurses would advise appropriate infection control measures as described previously. ${ }^{11,15-17}$ Briefly, patients were placed in single-room isolation with strict contact precautions. Cleaning staff was specifically trained to perform meticulous disinfection of high-touch areas, such as bedrail, bedside table, and locker. ${ }^{18}$ Terminal cleansing of patients' rooms and change of curtains were performed during their discharge or transfer.

Epidemiologic characteristics of all patients with newly identified CPE were retrieved from the clinical management system, which is a computer database with patients' information accessible from all public hospitals in Hong Kong. Risk factors for gastrointestinal colonization of CPE were investigated by case-control analysis. Two consecutive patients before and after a CPE case with negative result of CRE and CPE were chosen as controls. Nosocomial onset of CPE was defined as a positive culture of CPE identified by opportunistic screening performed after 48 hours of hospitalization, or safety net screening on the 14th day of hospitalization, whereas nosocomial acquisition of $\mathrm{CPE}$ was defined as secondary $\mathrm{CPE}$ cases identified during contact tracing. Outbreak investigations were conducted when 3 or more epidemiologically linked patients (same ward) with nosocomial onset or acquisition of CPE was identified. This study was approved by the institutional review board of the University of Hong Kong/Hospital Authority Hong Kong West Hospital Cluster.

\section{Bacterial Identification and Susceptibility Testing}

CPE screening was performed as previously reported. ${ }^{19,20}$ Briefly, a stool or rectal swab with visible fecal staining was submerged into brain heart infusion broth supplemented with $10 \mathrm{mg} / \mathrm{L}$ vancomycin (Sigma Aldrich) and $0.5 \mathrm{mg} / \mathrm{L}$ meropenem (Hospira), incubated at $37^{\circ} \mathrm{C}$ overnight for enrichment. Ten microliters of the enriched broth was subcultured onto MacConkey agar with $2 \mu \mathrm{g} / \mathrm{mL}$ meropenem and incubated aerobically at $35^{\circ} \mathrm{C}$ for 48 hours. Enterobacteriaceae that grew on the selective agars were identified using matrixassisted laser desorption ionization-time of flight mass spectrometry (Bruker Daltonics). Antimicrobial susceptibilities of the Enterobacteriaceae were determined using the Kirby-Bauer disk diffusion method in accordance with the Clinical and Laboratory Standards Institute or manufacturer's instructions. The presence of carbapenemase in the isolates was screened using the combined-disc test (meropenem/imipenem/ertapenem alone vs combination with phenyl boronic acid or ethylenediaminetetraacetic acid) and multiplex polymerase chain reaction targeting $b l a_{\mathrm{NDM}}, b l a_{\mathrm{KPC}}$, and $b l a_{\mathrm{OXA}}, b l a_{\mathrm{IMP}}$, $b l a_{\mathrm{VIM}}, b l a_{\mathrm{GES}}, b l a_{\mathrm{IMI}}$, and $b l a_{\mathrm{SME}}$ genes. ${ }^{19}$

\section{Statistical Analysis}

A logistic regression model was used to determine the risk factors of gastrointestinal colonization of $\mathrm{CPE}$. An odds ratio significantly greater than 1 implied higher risk of having gastrointestinal colonization of CPE. Cox regression was used to analyze risk factors of persistent colonization of CPE. CPE clearance was defined as the event. A hazard ratio of less than 1 implied longer duration of CPE colonization. Both bivariable and multivariable analyses were conducted. Multivariable analyses began with the full model and backward elimination was applied to select the final model. SPSS, version 20 (IBM), was used to perform the statistical analyses. A level of significance at $5 \%$ was adopted.

\section{RES ULTS}

From July 1, 2011, through December 31, 2015, a total of 66,672 fecal specimens from 31,526 patients were screened in our healthcare network, of which 1,374 specimens $(2.1 \%)$ from 518 patients $(1.6 \%)$ were positive for CRE, and 345 
specimens $(0.5 \%)$ from 100 patients $(0.3 \%)$ were positive for CPE (Figure 1). The overall prevalence of CRE and CPE was 15.0 and 2.9 per 100,000 patient-days, respectively. The number and prevalence (per 100,000 patient-days) of CRE increased from 17 (2.2) in 2012 to 268 (33.9) in 2015 $(P<.001)$, whereas the corresponding figures of $\mathrm{CPE}$ increased from $2(0.3)$ to $63(8.0)(P<.001)$, respectively. Of the 100 patients newly identified with gastrointestinal colonization of CPE, there were 69 males with a median (range) age of 60 (1-97) years. There were 48 medical patients (48\%), 31 surgical patients, 10 orthopedic patients, 5 pediatric patients, 2 oncology patients, and another 4 patients who stayed in the adult intensive care unit. Thirty-seven (37\%) of 100 patients had nosocomial onset of CPE with a median (range) of 15 (3-40) days after hospitalization. Twenty-one $(56.8 \%)$ of the 37 patients with nosocomial onset of CPE were identified from opportunistic and safety net screening from 17 different wards; the remaining 16 (43.2\%) had nosocomial acquisition of CPE identified as secondary cases during contact tracing in 14 wards. There was no evidence of clustering of cases to suggest hospital outbreaks. The risk factors for gastrointestinal colonization with CPE are shown in Table 1 . The presence of wound or drain and the use of cephalosporins, carbapenems, and PPIs in the preceding 6 months were independent risk factors in the multivariable analysis.

More than 1 specimen was received from 79 patients for $\mathrm{CPE}$ screening, with the median (range) follow-up time of 33 (3-545) days. Spontaneous clearance of CPE was noted in $57(72.2 \%)$ of 79 patients, with a median (range) of $30(3-411)$ days during our study period. Consumption of both antibiotics and PPIs after CPE identification was associated with later clearance of CPE (hazard ratio, 0.35 [95\% CI, 0.17-0.73]) (Table 2), compared with patients without use of both antibiotics and PPIs. On the other hand, the use of both antibiotics and PPIs in the 6 months preceding CPE identification was not significantly associated with the duration of clearance of CPE
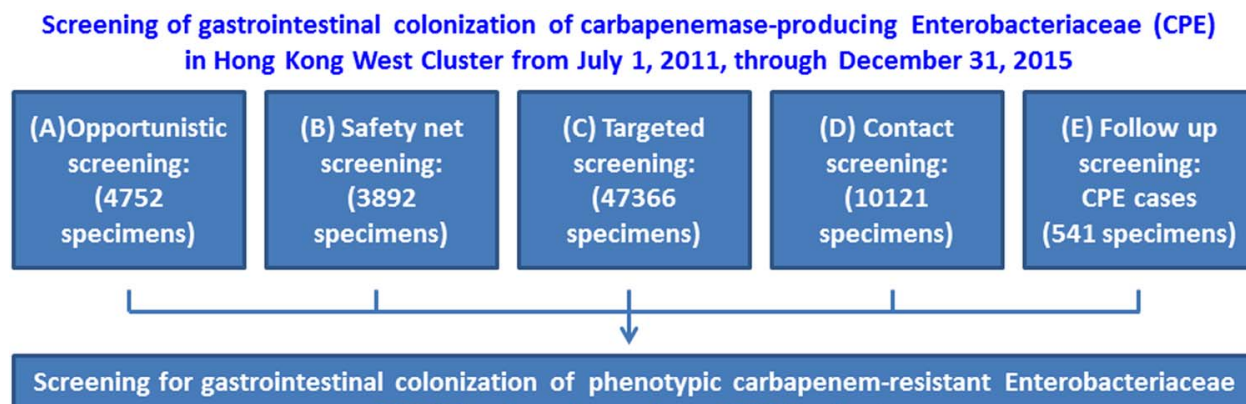
66672 fecal specimens from 31526 patients

$\downarrow$

Gastrointestinal colonization of phenotypic carbapenem-resistant Enterobacteriaceae 1374 / 66672 (2.1\%) fecal specimens from 518 / $31526(1.6 \%)$ patients

Gastrointestinal colonization of carbapenemase-producing Enterobacteriaceae (CPE) 345 / 66672 (0.5\%) fecal specimens from 100 / 31526 (0.3\%) patients

$\downarrow$

Yield of detection of gastrointestinal colonization of CPE by different screening approaches

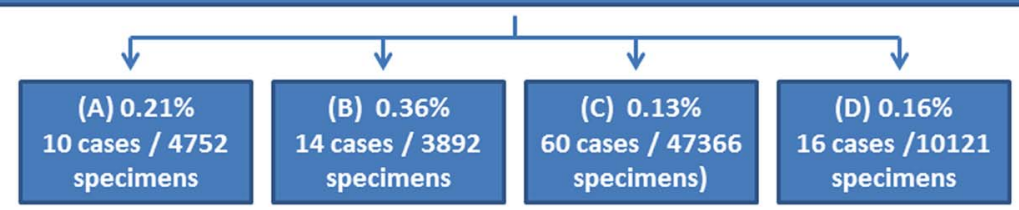

FIGURE 1. Screening of gastrointestinal colonization of carbapenemase-producing Enterobacteriaceae (CPE) in Hong Kong West Cluster (July 1, 2011, through December 31, 2015). Target admission screening includes (i) "Whom TO screen" criteria, where T represents a history of medical "tourism" with hospitalization outside Hong Kong and O represents a history of receiving surgical operation outside Hong Kong within 12 months preceding admission, (ii) patients with histories of admissions to local hospitals in the past 3 months, and (iii) patients being admitted to high-risk areas, including intensive care unit, liver transplant unit, hematology unit, and renal dialysis unit. Opportunistic screening for CPE is added on all fecal specimens requested for Clostridium difficile culture or cytotoxin assay, whereas safety net screening represents testing for CPE in all patients on the 14th day of hospitalization. Extensive contact tracing for secondary cases was performed when a CPE-carrying index patient was identified. Extensive contact tracing included all potentially exposed patients during the entire period of hospitalization of the index patient. Of 63 patients with CPE diagnosed by targeted screening, 26 (41\%) had history of hospitalization outside Hong Kong in the preceding 12 months (24 from China, 1 from Thailand, and 1 from India), whereas 34 (54\%) had history of hospitalization in Hong Kong in the preceding 3 months. The remaining 3 patients had no history of hospitalization and had probably acquired CPE in the community from an unknown source. 
TABLE 1. Risk Factors for Gastrointestinal Colonization of Carbapenemase-Producing Enterobacteriaceae (CPE)

\begin{tabular}{|c|c|c|c|c|c|c|}
\hline \multirow[b]{2}{*}{ Characteristic } & \multirow[b]{2}{*}{$\begin{array}{l}\text { Patients with gastrointestinal } \\
\text { colonization of CPE }(n=100)\end{array}$} & \multirow[b]{2}{*}{$\begin{array}{l}\text { Patients without gastrointestinal } \\
\text { colonization of CPE }(n=400)\end{array}$} & \multicolumn{2}{|c|}{ Bivariable analysis } & \multicolumn{2}{|c|}{ Multivariable analysis $^{\mathrm{a}}$} \\
\hline & & & $\begin{array}{l}\text { Odds ratio } \\
(95 \% \mathrm{CI})\end{array}$ & $P$ value & Odds ratio $(95 \% \mathrm{CI})$ & $P$ value \\
\hline Age, mean $\pm S D, y$ & $59.4 \pm 20.8$ & $58.9 \pm 24.3$ & $1.00(0.99-1.01)$ & .865 & & \\
\hline Male sex & $69(69.0)$ & $213(53.3)$ & $1.95(1.23-3.12)$ & .005 & $1.91(1.15-3.18)$ & .013 \\
\hline Resident in long-term care facilities & $7(7.0)$ & $35(8.8)$ & $0.79(0.34-1.82)$ & .573 & & \\
\hline \multicolumn{7}{|l|}{ Presence of indwelling device } \\
\hline Nasogastric tube & $11(11.0)$ & $19(4.8)$ & $2.48(1.14-5.39)$ & .022 & & \\
\hline Tracheostomy or endotracheal tube & $9(9.0)$ & $8(2.0)$ & $4.85(1.82-12.90)$ & .002 & & \\
\hline Urinary catheter & $33(33.0)$ & $90(22.5)$ & $1.70(1.05-2.74)$ & .030 & & \\
\hline Wound or drain & $29(29.0)$ & $36(9.0)$ & $4.13(2.38-7.17)$ & $<.001$ & $3.12(1.70-5.71)$ & $<.001$ \\
\hline Presence of chronic diseases ${ }^{\mathrm{b}}$ & $50(50.0)$ & $196(49.0)$ & $1.04(0.67-1.61)$ & .858 & & \\
\hline \multicolumn{7}{|l|}{ Use of antibiotics in preceding 6 months ${ }^{c}$} \\
\hline Beta-lactam/ beta-lactamase inhibitors & $39(39.0)$ & $58(14.5)$ & $3.77(2.31-6.15)$ & $<.001$ & & \\
\hline Cephalosporins & $20(20.0)$ & $17(4.3)$ & $5.63(2.83-11.23)$ & $<.001$ & $3.06(1.42-6.59)$ & .004 \\
\hline Carbapenems & $22(22.0)$ & $26(6.5)$ & $4.06(2.19-7.53)$ & $<.001$ & $2.21(1.10-4.48)$ & .027 \\
\hline Fluoroquinolones & $12(12.0)$ & $23(5.8)$ & $2.24(1.07-4.66)$ & .032 & & \\
\hline $\begin{array}{l}\text { Use of proton pump inhibitors in } \\
\text { preceding } 6 \text { months }{ }^{\mathrm{d}}\end{array}$ & $60(60)$ & $105(26.3)$ & $4.21(2.67-6.66)$ & $<.001$ & $2.84(1.72-4.71)$ & $<.001$ \\
\hline History of hospitalization in past 6 months & $80(80.0)$ & $278(69.5)$ & $1.76(1.03-2.99)$ & .039 & & \\
\hline
\end{tabular}

NOTE. Data are no. (\%) unless otherwise specified.

${ }^{\mathrm{a}}$ Final model was selected by backward selection procedure with the full model including all the variables examined in the bivariable analysis. The Hosmer-Lemeshow goodness-of-fit statistics $=0.188, P=.996$.

${ }^{\mathrm{b} C h r o n i c ~ d i s e a s e s ~ i n c l u d e ~ c h r o n i c ~ c e r e b r a l ~ c o n d i t i o n s, ~ c h r o n i c ~ c a r d i o p u l m o n a r y ~ c o n d i t i o n s, ~ c h r o n i c ~ r e n a l ~ f a i l u r e, ~ l i v e r ~ c i r r h o s i s, ~ d i a b e t e s ~ m e l l i t u s, ~ a n d ~ m a l i g n a n t ~ t u m o r s . ~}$

${ }^{\mathrm{c}}$ Antibiotic use variables were assessed as binary variables, instead of quantitative variables. In usual circumstances, using variables in a quantitative manner (ie, taking the duration of exposure into account) would be more informative. Unfortunately, there was overrepresentation of zeros in the variables, such that using the variables as quantitative variables in the logistic regression model resulted in an unacceptable goodness-of-fit. With binary variables, it could be possible that true differences between the case and control groups were not detected or were greater than what was detected.

${ }^{\mathrm{d}}$ The proton pump inhibitors included dexlansoprazole, esomeprazole, lansoprazole, omeprazole, pantoprazole, and rabeprazole. 
TAвLE 2. Hazard Ratios (HRs) for Clearance of Carbapenemase-Producing Enterobacteriaceae (CPE) by Epidemiologic Characteristics of 79 Patients With $>1$ Specimen Requested for CPE Screening

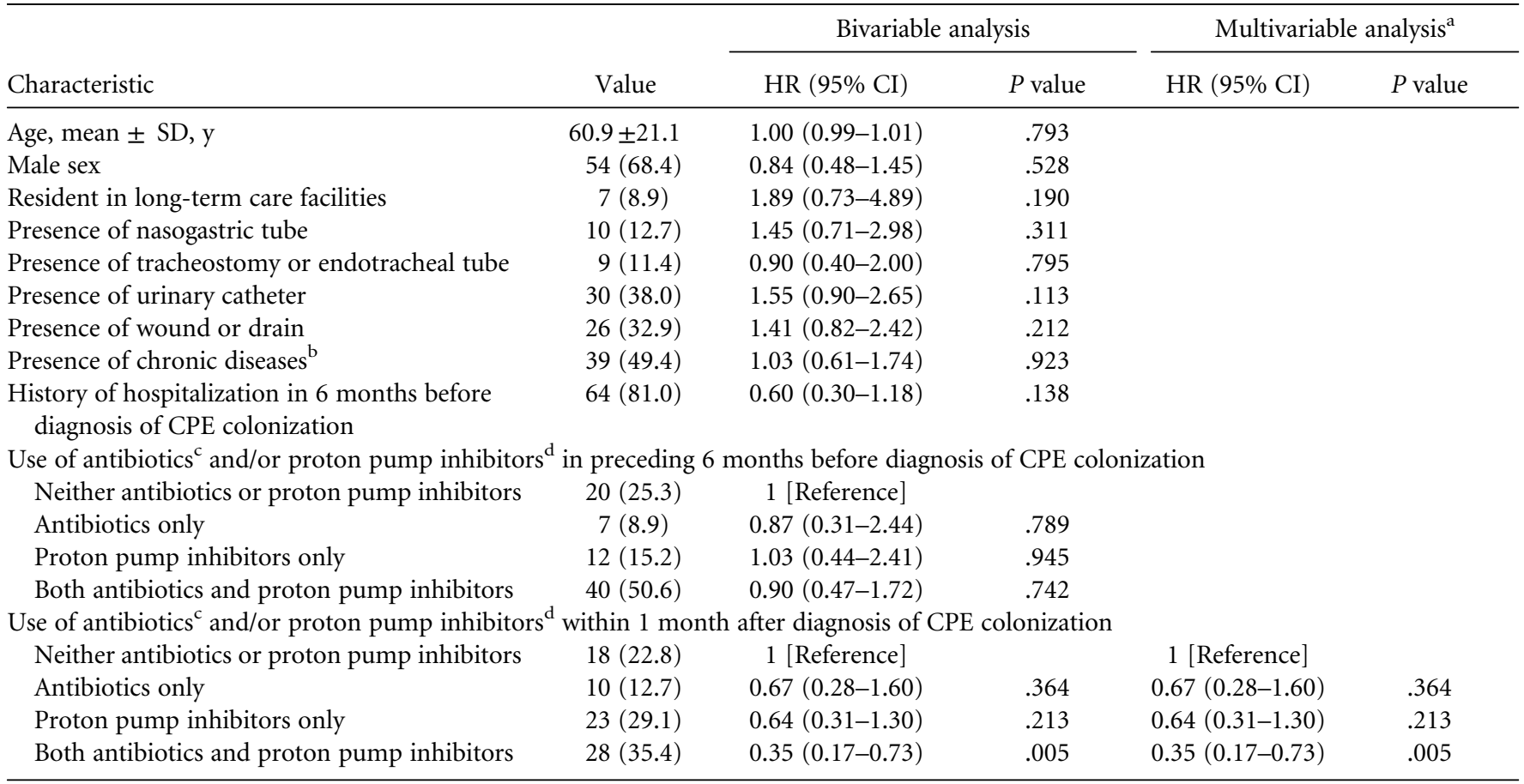

NOTE. Data are no. (\%) unless otherwise specified.

${ }^{a}$ Final model was selected by backward selection procedure with the full model including all the variables examined in the bivariable analysis, and only 1 variable was retained in the final model.

${ }^{\mathrm{b}}$ Chronic diseases include chronic cerebral conditions, chronic cardiopulmonary conditions, chronic renal failure, liver cirrhosis, diabetes mellitus, and malignant tumors.

${ }^{c}$ The antibiotics included beta-lactam/ beta-lactamase inhibitors, cephalosporins, carbapenems, and fluoroquinolones.

${ }^{\mathrm{d}}$ The proton pump inhibitors included dexlansoprazole, esomeprazole, lansoprazole, omeprazole, pantoprazole, and rabeprazole.

$(P=.742)$. The cumulative distribution plot of clearance of CPE patients with and without use of antibiotics and/or PPIs is illustrated in Figure 2.

Of the 100 patients with gastrointestinal colonization of CPE, $10(10 \%)$ had isolation of CPE from clinical specimens, after a median (range) duration of 55 (1-482) days. Six patients had CPE isolated in urine, 1 in bile, 1 in sputum, 1 in wound, and 1 in both blood and sputum specimens. Nine (9\%) of the 100 patients died during the same episode of hospitalization in which CPE was identified.

Molecular characterization of the 100 first isolates of patient-unique CPE strains is illustrated in Table 3. One patient had multiple NDM-producing Enterobacteriaceae isolates, including Escherichia coli, Salmonella species, and Proteus mirabilis, during the course of hospitalization as we reported previously. ${ }^{20}$

\section{I S C U S S I O N}

The overall prevalence of gastrointestinal colonization of CRE $(1.6 \%)$ in our hospital network was much lower than that reported in previous studies conducted in the healthcare facilities in the United States $(5.4 \%),{ }^{21}$ mainland China $(6.6 \%),{ }^{22}$ and Israel $(12.0 \%) .{ }^{23}$ However, the prevalence of CRE and CPE increased significantly between 2012 and 2015, by 15- and 30 -fold, respectively. More than $20 \%$ of the patients with CRE isolates had carbapenemase-producing strains in 2015, which was twice that reported in our group's previous study. ${ }^{19}$ Multiple sources of $\mathrm{CPE}$ acquisition were noted in our cohort. Approximately $25 \%$ of new cases had a history of hospitalization outside Hong Kong, especially in mainland China, where $\mathrm{CPE}$ was recently reported in both metropolitan and rural healthcare settings. ${ }^{24,25}$ The route of acquisition in most patients was uncertain, except for a minority with clear epidemiologic link suggestive of nosocomial transmission during the extensive contact tracing exercise as previously described. ${ }^{11,26}$ The possibility of community acquisition could not be excluded. Carbapenemase-producing microorganisms have been reported in food-producing animals and food products. ${ }^{27,28}$ Avoidance of undercooked food items and vigilant attention to kitchen hygiene to prevent cross-contamination are advisable to reduce the risk of acquisition of multidrug-resistant organisms. ${ }^{29}$ 


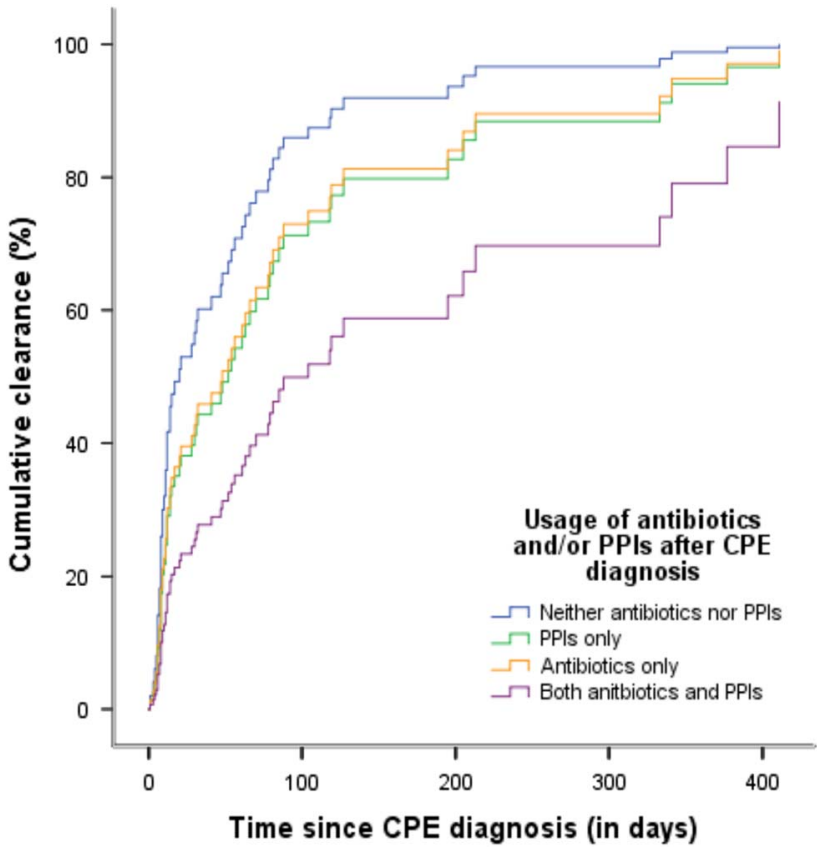

FIGURE 2. Cumulative clearance of carbapenemase-producing Enterobacteriaceae (CPE) patients with use of antibiotics and proton pump inhibitors (PPIs). Data on the use of PPIs and antibiotics without activities against CPE in patients after diagnosis of CPE gastrointestinal colonization.

ta вLE 3. Molecular Characteristics of the First Isolate of PatientUnique Strain of Carbapenemase-Producing Enterobacteriaceae Isolated From 100 Patients

\begin{tabular}{llc}
\hline Type of $\beta$-lactamase & Organisms & Number \\
\hline $\begin{array}{l}\text { New Delhi metallo- } \beta \text {-lactamase } \\
\text { (NDM) }\end{array}$ & Escherichia coli & 56 \\
& $\begin{array}{l}\text { Klebsiella } \\
\text { pneumoniae }\end{array}$ & 10 \\
& $\begin{array}{l}\text { Enterobacter } \\
\text { aerogenes }\end{array}$ & 2 \\
& Enterobacter cloacae & 2 \\
& Citrobacter koseri & 1 \\
& Citrobacter freundii & 1 \\
& Raoultella species & 1 \\
& K. pneumoniae & 20 \\
K. pneumoniae carbapenemase (KPC) & Enterobacter species & 1 \\
Metallo- $\beta$-lactamase (IMP) & K. pneumoniae & 4 \\
& E. cloacae & 1 \\
Oxacillinase metallo- $\beta$-lactamase & E. coli & 1 \\
(OXA) &
\end{tabular}

Opportunistic and safety net screening detected a significant proportion of CPE cases who were not colonized at the time of admission but acquired CPE during hospitalization. Broad-spectrum antibiotics were not infrequently used in patients with prolonged hospitalization. These screening strategies facilitated the identification of $\mathrm{CPE}$ carriage in patients with low microbial load upon admission, which was similar to the detection of MRSA in our group's previous study. ${ }^{30}$ In fact, use of broad-spectrum antibiotics such as cephalosporins and carbapenems was significantly associated with $\mathrm{CPE}$ acquisition in the multivariable analysis, similar to findings in previous studies of gastrointestinal colonization of CPE during active surveillance cultures. ${ }^{31,32}$ Use of broad-spectrum antibiotics without intrinsic activity against CPE contributed to prolonged colonization. The duration of gastrointestinal colonization of CPE varied from months to years. After a large, single-center outbreak of KPC-producing Klebsiella pneumoniae in Germany, most patients had spontaneous decolonization within 6 months after acquisition, but long-term carriage of more than 3 years was possible. ${ }^{33}$

To the best of our knowledge, our study was the first to demonstrate that use of PPIs was a risk factor for CPE acquisition. In addition, the combination of broad-spectrum antibiotics and PPI consumption further delayed CPE clearance. PPIs were previously shown to alter the composition of the gut microbiota by lowering the abundance of gut commensals, reducing microbial diversity, ${ }^{34}$ and encouraging bacterial overgrowth in the small intestine, resulting in an overall increased risk of recurrent aspiration pneumonia in the elderly $^{35}$ and C. difficile-associated diarrhea. ${ }^{36}$ Concomitant use of antibiotics and PPIs also conferred a greater risk of C. difficile infection. ${ }^{37}$ Although the mechanism of PPI use and gastrointestinal colonization of $\mathrm{CPE}$ remains uncertain, hypochlorhydria induced by PPIs ${ }^{38}$ may have allowed the passage of CPE from oral intake, nasogastric tube, or percutaneous gastrostomy feeding to the lower intestine with colonization during hospitalization. Thus, it is conceivable that not just antibiotics but also PPIs may increase the microbial load of CPE within the gastrointestinal tract of these patients and prolong the duration of CPE carriage. Therefore, directly observed patient hand hygiene before meals and medications among hospitalized patients, especially in patients receiving concurrent broad-spectrum antibiotics and PPIs, is important because it reduces the risk of oral ingestion of CPE. Similarly, healthcare workers should comply with hand hygiene practice before preparing feeds for patients with nasogastric or percutaneous gastrostomy. Male patients were shown to be significantly associated with CPE carriage, possibly related to the gender difference in hand hygiene practice, in which females washed their hands significantly more often than males. ${ }^{39}$ Results from this study supported the need for limiting use of broad-spectrum antibiotics and PPIs in patients with gastrointestinal colonization with $\mathrm{CPE}$ to facilitate spontaneous clearance. Whether histamine $\mathrm{H}-2$ receptor blocker, which has less profound acid suppression, would have lesser effect on CPE colonization requires further investigation. Although we hypothesized that PPI use could be associated with CPE acquisition and colonization, a previous study has not found any association between PPIs and CRE acquisition. ${ }^{21}$ Further study of this potential risk factor is warranted. 
There are several limitations of this study. First, the relatively small sample size may be inadequate to demonstrate other significant associations-for instance, the use of either antibiotics or PPIs after CPE identification on the duration of $\mathrm{CPE}$ colonization. Second, our strains of CPE were heterogeneous, comprising NDM-, KPC-, IMP-, and OXAproducing Enterobacteriaceae. However, we believe that the analysis of the clinical epidemiology in this group of patients provides useful information for the formulation of infection control policy, regardless of the actual molecular type of CPE. In summary, compliance with infection control and hand hygiene is particularly important for relevant healthcare workers and patients on concomitant treatment with antibiotics and PPIs, which predispose them to a more prolonged carriage of CPE.

\section{A C KNOWLEDGMENTS}

Financial support. Health and Medical Research Fund, Food and Health Bureau, Hong Kong SAR Government (ref. no. HKM-15-M12).

Potential conflicts of interest. All authors report no conflicts of interest relevant to this article.

Address correspondence to Kwok-Yung Yuen, State Key Laboratory of Emerging Infectious Diseases, Carol Yu Centre for Infection, Department of Microbiology, Queen Mary Hospital, The University of Hong Kong, Hong Kong Special Administrative Region, China (kyyuen@hku.hk)

\section{REFERENCES}

1. Tzouvelekis LS, Markogiannakis A, Psichogiou M, Tassios PT, Daikos GL. Carbapenemases in Klebsiella pneumoniae and other Enterobacteriaceae: an evolving crisis of global dimensions. Clin Microbiol Rev 2012;25:682-707.

2. Villegas MV, Pallares CJ, Escandon-Vargas K, et al. Characterization and clinical impact of bloodstream infection caused by carbapenemase-producing Enterobacteriaceae in seven Latin American countries. PLOS ONE 2016;11:e0154092.

3. Won SY, Munoz-Price LS, Lolans K, et al. Emergence and rapid regional spread of Klebsiella pneumoniae carbapenemaseproducing Enterobacteriaceae. Clin Infect Dis 2011;53:532-540.

4. Morris D, Boyle F, Morris C, et al. Inter-hospital outbreak of Klebsiella pneumoniae producing KPC-2 carbapenemase in Ireland. J Antimicrob Chemother 2012;67:2367-2372.

5. Weterings V, Zhou K, Rossen JW, et al. An outbreak of colistinresistant Klebsiella pneumoniae carbapenemase-producing Klebsiella pneumoniae in the Netherlands (July to December 2013), with inter-institutional spread. Eur J Clin Microbiol Infect Dis 2015;34:1647-1655.

6. Delory T, Seringe E, Antoniotti G, et al. Prolonged delay for controlling KPC-2-producing Klebsiella pneumoniae outbreak: the role of clinical management. Am J Infect Control 2015;43:1070-1075.

7. Schwaber MJ, Lev B, Israeli A, et al. Containment of a countrywide outbreak of carbapenem-resistant Klebsiella pneumoniae in Israeli hospitals via a nationally implemented intervention. Clin Infect Dis 2011;52:848-855.

8. Hayden MK, Lin MY, Lolans K, et al. Prevention of colonization and infection by Klebsiella pneumoniae carbapenemase- producing Enterobacteriaceae in long-term acute-care hospitals. Clin Infect Dis 2015;60:1153-1161.

9. Johnson AP, Woodford N. Global spread of antibiotic resistance: the example of New Delhi metallo-beta-lactamase (NDM)-mediated carbapenem resistance. J Med Microbiol 2013;62:499-513.

10. Ho PL, Li Z, Lai EL, Chiu SS, Cheng VC. Emergence of NDM1-producing Enterobacteriaceae in China. J Antimicrob Chemother 2012;67:1553-1555.

11. Cheng VC, Chan JF, Wong SC, et al. Proactive infection control measures to prevent nosocomial transmission of carbapenemresistant Enterobacteriaceae in a non-endemic area. Chin Med J (Engl) 2013;126:4504-4509.

12. Rossini A, Di Santo SG, Libori MF, Tiracchia V, Balice MP, Salvia A. Risk factors for carbapenemase-producing Enterobacteriaceae colonization of asymptomatic carriers on admission to an Italian rehabilitation hospital. J Hosp Infect 2016;92:78-81.

13. Cheng VC, Tai JW, Chen JH, et al. Proactive infection control measures to prevent nosocomial transmission of vancomycinresistant enterococci in Hong Kong. J Formos Med Assoc 2014; 113:734-741.

14. Cheng VC, Chen JH, So SY, et al. Use of fluoroquinolones is the single most important risk factor for the high bacterial load in patients with nasal and gastrointestinal colonization by multidrug-resistant Acinetobacter baumannii. Eur J Clin Microbiol Infect Dis 2015;34:2359-2366.

15. Cheng VC, Chen JH, Poon RW, et al. Control of hospital endemicity of multiple-drug-resistant Acinetobacter baumannii ST457 with directly observed hand hygiene. Eur J Clin Microbiol Infect Dis 2015;34:713-718.

16. Cheng VC, Tai JW, Wong LM, et al. Effect of proactive infection control measures on benchmarked rate of hospital outbreaks: an analysis of public hospitals in Hong Kong over 5 years. Am J Infect Control 2015;43:965-970.

17. Cheng VC, Tai JW, Li WS, et al. Implementation of directly observed patient hand hygiene for hospitalized patients by hand hygiene ambassadors in Hong Kong. Am J Infect Control 2016;44:621-624.

18. Cheng VC, Chau PH, Lee WM, et al. Hand-touch contact assessment of high-touch and mutual-touch surfaces among healthcare workers, patients, and visitors. J Hosp Infect 2015;90:220-225.

19. Ho PL, Cheung YY, Wang Y, et al. Characterization of carbapenem-resistant Escherichia coli and Klebsiella pneumoniae from a healthcare region in Hong Kong. Eur J Clin Microbiol Infect Dis 2016;35:379-385.

20. Cheng VC, Chen JH, Wong SC, Ho PL, Yuen KY. Gastrointestinal colonization with multiple New Delhi metallobeta-lactamase-producing Enterobacteriaceae isolates in the same patient: a potential challenge in outbreak investigation. J Hosp Infect 2016;92:108-109.

21. Swaminathan M, Sharma S, Poliansky Blash S, et al. Prevalence and risk factors for acquisition of carbapenem-resistant Enterobacteriaceae in the setting of endemicity. Infect Control Hosp Epidemiol 2013;34:809-817.

22. Zhao ZC, Xu XH, Liu MB, Wu J, Lin J, Li B. Fecal carriage of carbapenem-resistant Enterobacteriaceae in a Chinese university hospital. Am J Infect Control 2014;42:e61-e64.

23. Ben-David D, Masarwa S, Navon-Venezia S, et al. Carbapenemresistant Klebsiella pneumoniae in post-acute-care facilities in Israel. Infect Control Hosp Epidemiol 2011;32:845-853. 
24. Yang J, Ye L, Guo L, et al. A nosocomial outbreak of KPC-2producing Klebsiella pneumoniae in a Chinese hospital: dissemination of ST11 and emergence of ST37, ST392 and ST395. Clin Microbiol Infect 2013;19:E509-E515.

25. Zheng R, Zhang Q, Guo Y, et al. Outbreak of plasmid-mediated NDM-1-producing Klebsiella pneumoniae ST105 among neonatal patients in Yunnan, China. Ann Clin Microbiol Antimicrob 2016;15:10.

26. Cheng VC, Tai JW, Ng ML, et al. Extensive contact tracing and screening to control the spread of vancomycin-resistant Enterococcus faecium ST414 in Hong Kong. Chin Med J (Engl) 2012;125:3450-3457.

27. Guerra B, Fischer J, Helmuth R. An emerging public health problem: acquired carbapenemase-producing microorganisms are present in food-producing animals, their environment, companion animals and wild birds. Vet Microbiol 2014;171:290-297.

28. Morrison BJ, Rubin JE. Carbapenemase producing bacteria in the food supply escaping detection. PLoS One 2015;10: e0126717.

29. Cheng VC, Wong SC, Ho PL, Yuen KY. Strategic measures for the control of surging antimicrobial resistance in Hong Kong and mainland of China. Emerg Microbes Infect 2015;4:e8.

30. Cheng VC, Li IW, Wu AK, et al. Effect of antibiotics on the bacterial load of meticillin-resistant Staphylococcus aureus colonisation in anterior nares. J Hosp Infect 2008;70:27-34.

31. Papadimitriou-Olivgeris M, Marangos M, Fligou F, et al. Risk factors for KPC-producing Klebsiella pneumoniae enteric colonization upon ICU admission. J Antimicrob Chemother 2012;67:2976-2981.
32. Gagliotti C, Giordani S, Ciccarese V, et al. Risk factors for colonization with carbapenemase-producing Klebsiella pneumoniae in hospital: a matched case-control study. Am J Infect Control 2014;42:1006-1008.

33. Lubbert C, Lippmann N, Busch T, et al. Long-term carriage of Klebsiella pneumoniae carbapenemase-2-producing K pneumoniae after a large single-center outbreak in Germany. Am J Infect Control 2014;42:376-380.

34. Jackson MA, Goodrich JK, Maxan ME, et al. Proton pump inhibitors alter the composition of the gut microbiota. Gut 2016;65:749-756.

35. Dang TT, Majumdar SR, Marrie TJ, Eurich DT. Recurrent pneumonia: a review with focus on clinical epidemiology and modifiable risk factors in elderly patients. Drugs Aging 2015;32:13-19.

36. Janarthanan S, Ditah I, Adler DG, Ehrinpreis MN. Clostridium difficile-associated diarrhea and proton pump inhibitor therapy: a meta-analysis. Am J Gastroenterol 2012;107:1001-1010.

37. Kwok CS, Arthur AK, Anibueze CI, Singh S, Cavallazzi R, Loke YK. Risk of Clostridium difficile infection with acid suppressing drugs and antibiotics: meta-analysis. Am J Gastroenterol 2012;107:1011-1019.

38. Corleto VD, Festa S, Di Giulio E, Annibale B. Proton pump inhibitor therapy and potential long-term harm. Curr Opin Endocrinol Diabetes Obes 2014;21:3-8.

39. Fung IC, Cairncross S. How often do you wash your hands? A review of studies of hand-washing practices in the community during and after the SARS outbreak in 2003. Int J Environ Health Res 2007;17:161-183. 